\title{
6 Von der „Pressemitteilung“ zur „An-alle-Mitteilung": Der Medien-Doktor PR Watch an der TU Dortmund
}

Marcus Anhäuser und Holger Wormer

- Pressemitteilungen beeinflussen die Qualität journalistischer Beiträge und informieren zugleich nicht mehr nur Journalisten, sondern die breite Öffentlichkeit direkt, wodurch eine besondere Verantwortung insbesondere bei Gesundheitsthemen entsteht.

- Das Medien-Doktor-Projekt bietet Kriterien an, die sich dazu eignen, Fallstricke und Probleme in der Qualität von Pressemitteilungen zu offenbaren, und die zugleich eine Hilfestellung bei der Erstellung solcher Texte sein können.

- Der Medien-Doktor bewertet mit diesen Kriterien neben journalistischen Beiträgen regelmäßig Pressemitteilungen und präsentiert diese auf seiner Webseite.

- Die Kriterien des Medien-Doktor diskutieren wir ausgiebig mit Vertretern der Wissenschafts-PR, um sie weiter zu entwickeln und so das Bewusstsein für die Qualität in diesem Bereich zu schärfen.

Fiona Fox beschlich beim Nachdenken über den Einfluss von Pressemitteilungen auf die Medien ein ungutes Gefühl: Die Direktorin des britischen Science Media Centre (SMC), das Journalisten in der Wissenschaftsberichterstattung unterstützt, schrieb im Februar 2012 (Fox 2012): „I have a horrible feeling that if we took the worst newspaper headlines and traced back their origins - we may find the press release was to blame in far more cases than any of us would care to admit." Sie wisse im übrigen nicht, ob das jemals jemand erforscht hätte. 
Wie es der Zufall so will, hatte just zehn Tage zuvor ein Team um die Medienforscher Lisa Schwartz und Steven Woloshin vom Dartmouth Institute for Health Policy and Clinical Practice in den USA genau zu dieser Fragestellung eine Studie im British Medical Journal (BMJ) veröffentlicht (Schwartz et al. 2012). Zentrales Ergebnis: Am Bauchgefühl von Fiona Fox könnte etwas dran sein.

Die amerikanische Gruppe hatte 68 Pressemitteilungen zu Fachartikeln aus fünf bedeutenden medizinischen Fachmagazinen gesammelt und untersucht, welchen Einfluss sie auf die journalistische Berichterstattung hatten. Ihr Ergebnis:

„Higher quality press releases issued by medical journals were associated with higher quality reporting in subsequent newspaper stories. [...] Fundamental information such as absolute risks, harms, and limitations was more likely to be reported in newspaper stories when this information appeared in a medical journal press release than when it was missing from the press release or if no press release was issued. Furthermore, our data suggest that poor quality press releases were worse than no press release being issued: fundamental information was less likely to be reported in newspaper stories when it was missing from the press release than where no press release was issued at all (although the findings were generally not statistically significant)."

Dass Pressemitteilungen die Arbeit von Journalisten beeinflussen, ist natürlich nicht überraschend. Angesichts schrumpfender Redaktionen, sinkender Honorare und weniger Zeit für Recherchen muss man indes einen zunehmenden Einfluss annehmen. Schon vor mehr als fünf Jahren räumte selbst ein gestandener Wissenschaftsredakteur wie Mark Henderson von der Londoner Times ein, dass er zwar „so weit wie möglich“ vermeide, sich allein auf Pressemitteilungen zu verlassen, aber „wenn es eine gute Pressemitteilung gibt und Du vier Geschichten am Tag zu schreiben hast, wirst Du diese Abkürzung nehmen"1.

Wie durchlässig der journalistische Filter im Gesundheitsbereich sein kann, zeigte auch ein Team um die Kommunikationswissenschaftlerin Doreen Reifegerste von der TU Dresden (Reifegerste et al. 2014): Anhand von rund 50 Pressemitteilungen einer Krankenkasse zum Thema Prävention, die es innerhalb eines Jahres in die Medien geschafft hatten, lasse sich feststellen, „dass ein Großteil [...] (66,8\%) von den Journalisten nahezu identisch übernommen wurde. [...] Nur bei 37\% der Artikel war eine Recherche aufgrund zusätzlich zur Pressemitteilung vorhandenen Informationen zu erkennen." Der Anteil der kopierten Pressemitteilungen lag demnach in regionalen Tageszeitungen bei rund 54\%, in Anzeigenblättern sogar bei fast $88 \%$.

Hinzu kommt, dass die Bezeichnung „Pressemitteilung“ ohnehin nur noch teilweise zutrifft; in Zeiten des Internets und anderer digitaler Kanäle müss-

1 Zitiert und übersetzt nach Brumfiel, Georg (2009): Supplanting the old media? Nature, vol. 458, S. 275 
6 Von der „Pressemitteilung“ zur „An-alle-Mitteilung“:

te man eigentlich von einer „An-alle-Mitteilung“ sprechen: Längst sind die PR-Mitteilungen von Hochschulen, Forschungsinstituten, -organisationen oder (Pharma-)Firmen, die in Prä-Internetzeiten fast exklusiv an Journalisten verschickt worden waren, für jeden online frei verfügbar - auf den Seiten und Kanälen der Einrichtungen oder auf Verteiler-Plattformen wie dem „Informationsdienst Wissenschaft“ (idw). Damit erreichen diese Artikel nicht mehr nur Medien-Profis, die - wenn sie ihren Beruf ernst nehmen - diese Medieninformationen einschätzen können, kritisch hinterfragen und weitere Meinungen einholen, bevor sie dann auf Basis der gesamten Recherche berichten. Stattdessen können Nachrichten aus der Wissenschafts- und Medizin-PR nun prinzipiell jeden erreichen, der nach Informationen zu einem Thema sucht, nicht nur Journalisten, sondern auch Medien- und Medizinlaien; eine einfache Google-Suche liefert neben Patienteninformationen (und im günstigen Fall Fachinformationen) und journalistischen Texten eben auch „Pressemitteilungen“ frei Haus für alle - und es bliebe zu testen, welchen Text ein Laie im Zweifel vertrauensvoller findet: den vielleicht aufgepeppten, journalistischen Artikel auf der Webseite einer Regionalzeitung oder den Pressetext auf der Klinikseite der Universitätskrankenhauses. Wissenschaftler und Mediziner und deren Institutionen genießen nach wie vor ein sehr hohes Vertrauen in der Bevölkerung, was man von Journalisten nicht unbedingt behaupten kann, wie Umfragen immer wieder bestätigen².

Beides zusammen, die zunehmend prekäre Situation im Journalismus und die direkte Verbreitung an mediale und medizinische Laien (Endnutzer) durch den freien Zugang im Internet, werfen endgültig die Frage auf, wie es um die Qualität solcher Pressemitteilungen bestellt ist. Und ob nicht eine neue Verantwortung für die zu „Endnutzerstellen“ gewandelten „Pressestellen“ entsteht, wenn diese sich nicht mehr darauf zurückziehen können, ihre Mitteilung diene ja nur als Basis für weitere Recherchen der Journalisten und dass diese eben zugespitzt werden müsse, um von hartgesottenen Redaktionen überhaupt beachtet zu werden.

Das ungute Gefühl, von dem Fiona Fox sprach, hatte angesichts all dieser Beobachtungen fast zeitgleich das Medien-Doktor-Team am Lehrstuhl Wissenschaftsjournalismus der TU Dortmund beschlichen. Nach einigen Vorbereitungen haben wir dann im Herbst 2012 ein entsprechendes BMBF-Projekt beantragt sowie im Mai 2013 in einem experimentellen Ansatz begonnen, mit dem „Medien-Doktor PR-Watch“" erste Pressemitteilungen aus dem Medizinund dem Umweltbereich so detailliert zu bewerten, wie wir es seit $2010 \mathrm{mit}$ journalistischen Beiträgen im bisherigen Medien-Doktor-Projekt tun. Seit

2 siehe etwa die Allensbacher Berufsprestige-Skala 2013 (http://www.ifd-allensbach.de/uploads/tx_reportsndocs/ PD_2013_05.pdf)

3 http://www.medien-doktor.de/pr-watch/ 
Juni 2015 werden journalistische Beiträge und Pressemitteilungen sogar im direkten Vergleich analysiert (s.u.).

Grundlage der Bewertung journalistischer Artikel sind dabei zehn „medizinjournalistische“ und drei „allgemeinjournalistische“ Kriterien (s. Textbox „13 Kriterien zur Bewertung medizinjournalistischer Berichterstattung“weiter unten). Die medizinjournalistischen Kriterien wurden ursprünglich entwickelt von australischen Journalisten und Medizinern bzw. Public HealthExperten und wurden weltweit in ähnlichen Projekten u.a. in Australien und den USA („Media-Doctor“, „HealthNewsReview“) erprobt. Anhand dieser zehn Basis-Kriterien hinterfragen wir, ob Leser, Zuschauer und Zuhörer in einem Beitrag die Informationen erhalten, die sie für ein ausreichendes Bild über eine Therapie, ein Medikament oder einen diagnostischen Test benötigen. Die drei weiteren Kriterien - die wir am Lehrstuhl Wissenschaftsjournalismus im Diskurs mit unseren Gutachtern aus Ergebnissen der journalistischen Qualitätsforschung abgeleitet haben - beschreiben die Qualität nach typisch journalistischen Maßstäben: Ist ein Thema relevant, aktuell und/oder ungewöhnlich? Stimmen die überprüfbaren Fakten? Ist das Thema verständlich und ansprechend dargestellt?

Nach unseren bisherigen Tests sind diese Kriterien - mit womöglich erstaunlich wenig Abstrichen - nicht nur dazu geeignet die Qualität eines journalistischen Beitrags zu bestimmen. Auch für Pressemitteilungen können sie eine Art Geländer sein, an dem sich Autoren entlang bewegen können. Dazu muss man noch lange nicht der Auffassung einzelner Vertreter der WissenschaftsPR folgen, dass diese ohnehin auch „Journalismus“ betriebe (woraus sogar unmittelbar folgte, dass die für den Medizinjournalismus etablierten Kriterien anwendbar sein müssten). Fundierte, transparente und verständliche Informationen können - so oder so - aber nicht nur Journalisten gebrauchen.

Inwieweit die Kriterien im Einzelnen für die Anforderungen in der Wissenschafts-PR angepasst werden müssen, evaluieren wir derzeit im erwähnten BMBF-geförderten Forschungsprojekt INKA ${ }^{4}$, in dem wir in Kooperation mit dem Deutschen Cochrane Zentrum Freiburg die gesamte Informationskaskade vom Fachartikel über die Pressemitteilung bis zum journalistischen Beitrag untersuchen. So erscheint es womöglich diskussionswürdig, ob man von Pressestellen verlangen soll, eine zweite Quelle jenseits der eigenen Institution zu zitieren, um etwa das präsentierte Ergebnis seiner Studie kritisch zu beleuchten. Was im Journalismus als Standard gilt, erscheint bei interessengeleiteter Kommunikation zuerst einmal ungewöhnlich, vielleicht sogar absurd. Andererseits: Auch in der Wissenschaft gehört es dazu, (evtl. sogar widersprüchliche) Resultate anderer zu erwähnen - warum soll man dann die seriöse Kommunikation dieser Wissenschaft davon ausnehmen? Andere

4 http://www.medien-doktor.de/medizin/specials/bmbf-projekt 
6 Von der „Pressemitteilung“ zur „An-alle-Mitteilung“:

der für journalistische Beiträge verwendeten Kriterien, etwa Angaben zur Finanzierung einer Studie oder mögliche Interessenkonflikte der beteiligten Mediziner, können für Pressemitteilungen ebenfalls als Standard diskutiert werden. Umso mehr, da es erste Hinweise gibt, dass auch Fachartikel der Ursprung für Übertreibungen (,Spin') und zu weitgehende Handlungsempfehlungen in der Kaskade vom Fachartikel über die Pressemitteilung bis zum journalistischen Artikel sind (Yavchits et al. 2012; Prasad et al. 2013).

Wie hilfreich bereits dieses Kriterienset bei der Bestimmung der Qualität von Pressemitteilungen sein kann, möchten wir an drei versuchsweisen Bewertungen medizinischer Pressemitteilungen demonstrieren, die wir im Rahmen unseres experimentell angelegten Teilprojekts „Medien-Doktor PRWatch"s zufällig ausgewählt hatten. Wir arbeiten hier Probleme dieser Texte heraus und zeigen, wie die verwendeten Kriterien helfen können, den Informationsgehalt von Texten zu verbessern.

\section{Pressemitteilung 1:}

„Heilung für bestimmte Leukämieform ohne Chemotherapie möglich. ${ }^{“ 6}$

Heilsversprechen gegen Krebs sind immer mit Vorsicht zu genießen. Autoren - ob nun Journalisten oder andere - tragen bei einer so gravierenden und angstbesetzten Erkrankung eine besondere Verantwortung. Trotzdem wagte die Pressestelle des Universitätsklinikums Ulm folgenden Titel für ihre Pressemitteilung: „Heilung für bestimmte Leukämieform ohne Chemotherapie möglich.“ Im Text heißt es dann: „98 Prozent der betroffenen Patienten können so ohne den Einsatz von Chemotherapie geheilt werden."

Immerhin versucht die Pressemitteilung damit auch, den möglichen Nutzen überhaupt in Zahlen zu fassen - und stellt klar, dass es sich nur um eine bestimmte Form der Leukämie handelt. Die Formulierung „98 Prozent [...] können geheilt werden" stellt den Nutzen allerdings übertrieben dar. Eine so abschließende Aussage ist auf der Basis der Studie, die Anlass der Pressemitteilung war, nicht möglich - und bei Überlebensraten zu Krebs so auch unüblich. Diese werden in der Regel mit einem Überlebenszeitraum (meist fünf Jahre) angegeben. In dieser Studie wurden die meisten Patienten aber nicht mal drei Jahre nachbeobachtet. In der Pressemitteilung fällt die Zeitangabe unter den Tisch. Der Heilungserfolg von „98 Prozent" schrumpft weiter, wenn man erfährt, dass in der Studie nur 77 Patienten behandelt wurden, und sich der Vorteil gegenüber der Vergleichsgruppe in absoluten Zahlen aus

5 http://www.medien-doktor.de/pr-watch

6 Bewertung: http://www.medien-doktor.de/pr-watch/2013/07/heilung-fur-bestimmte-leukamieform-ohnechemotherapie-moglich) 
nur wenigen Patienten ergibt. In der Fachpublikation selbst heißt es daher zurückhaltend: „[...] is at least not inferior and may be superior to [...]“.

Ärgerlich ist, dass in einem Text mit dem Wort Chemotherapie im Titel ein Aspekt wie Risiken und Nebenwirkungen gar nicht angesprochen wird. Und das, obwohl Chemotherapien in der Öffentlichkeit fast berüchtigt für Nebenwirkungen sind, und es in diesem Fall auch noch um ein Arsen(!)-Präparat in der neuen Therapie geht.

Andere Aspekte werden in dieser Pressemitteilung ebenfalls nicht ausreichend beleuchtet: Wenn schon „Heilung“ versprochen wird, möchte man als Betroffener wissen, wo und in welchem Rahmen? Nur an der Ulmer Uniklinik? Nur im Rahmen einer Studie für spezielle Patientengruppen? Was würde das kosten? Was würde die Krankenkasse übernehmen? Doch der Pressetext gibt dazu kaum Informationen.

Angesichts der Begriffskombination von „Leukämie“ und „Chemotherapie“ mit „Heilung“ erscheint es dann auf Seiten der Journalisten nicht überraschend, dass eine Reihe von Medien die Nachricht aufgegriffen hat. Interessanterweise formulieren einige dieser Medien - anders als oft zu befürchten zurückhaltender als das Universitätsklinikum selbst. So heißt es etwa bei der Online-Newsseite Hannover-Zeitung „Die neue Therapiekombination sei der bisherigen Standardtherapie mindestens nicht unterlegen, folgern die Wissenschaftler. " Menschen, die lediglich die Pressemitteilung im Internet finden, wird da deutlich mehr Hoffnung gemacht.

\section{Pressemitteilung 2:}

„Schwerkranke Patienten mit undichter Herzklappe profitieren von KatheterBehandlung"7

Eine Pressemitteilung der Universitätsklinik Heidelberg berichtet über eine kleine (rund drei [!] Monate zuvor online veröffentlichte) Studie, in der ein Verfahren gegen undichte Herzklappen bei alten Menschen mit Herzschwäche getestet wird. Dabei wird bei diesen Hochrisikopatienten mittels eines Katheters eine spezielle Klammer (MitraClip) bis zum Herzen geführt und damit die Herzklappe abgedichtet.

Den Nutzen des Verfahrens beschreibt der Pressetext zwar nicht in konkreten Zahlen, zieht dafür aber zahlreiche Parameter wie die Belastbarkeit der Patienten und den Hang zur Luftnot heran - und versucht den Nutzen für die Therapierten u.a. so einzuordnen: „Ihr Gesundheitszustand ist vergleichbar

7 Bewertung: http://www.medien-doktor.de/pr-watch/2013/10/schwerkranke-patienten-mit-undichter-herzklappeprofitieren-von-katheter-behandlung 
6 Von der „Pressemitteilung“ zur „An-alle-Mitteilung“:

mit Patienten, deren Mitralklappe im Rahmen eines chirurgischen Eingriffes repariert wurde."

Weniger hilfreich sind die Informationen zu Risiken und Nebenwirkungen. Statt die Ergebnisse nach einem Jahr zu berichten, wird - wie oft in Texten über „sanfte“ Katheter-Verfahren - herausgestellt, dass der Eingriff „ausgesprochen risikoarm" sei - was auf den ersten Blick auch zu stimmen scheint. Angaben dazu werden sogar beziffert, aber nur für den Zeitraum von einem Monat: „Das Verfahren erwies sich [...] als sehr sicher: Das Sterberisiko der schwerkranken Patienten durch den Eingriff lag nach 30 Tagen bei 2,9 Prozent im Vergleich zu rund 12 Prozent nach einer Operation am Herzen“. Das Problem (und hier zeigt sich wie wichtig eine Einordnung des Studiendesigns ist): Im Fachartikel weisen die Autoren auf die limitierte Aussagekraft ihrer Studie hin (nur insgesamt 59 Teilnehmer, keine Kontrollgruppe). Die Vergleichszahl zur Sterblichkeit von 12\% stammt folglich nicht aus dieser Untersuchung, sondern aus der Literatur. Dass nach einem Jahr 12 von 59 Patienten verstorben waren (also mehr als 20\%), wird im Pressetext ebenfalls nicht erwähnt. Dies muss bei hochbetagten Patienten zwar nicht an der Kathetermethode liegen, aber zumindest sollte deutlich werden, dass die Aussagen $\mathrm{zu}$ Risiken keineswegs gut gesichert sind. In der Pressemitteilung fällt das alles unter den Tisch, ebenso wie Informationen zur Finanzierung der Studie durch die Herstellerfirma des untersuchten Medizinproduktes.

Warum warten die betreffenden Wissenschaftskommunikatoren mit ihrer (Monate nach der Online-Veröffentlichung der Studie ohnehin nicht aktuellen) Pressemitteilung nicht wenigstens bis zur nächsten, vielleicht aussagekräftigeren Studie?

\section{Pressemitteilung 3:}

„Rettung bei plötzlichem Herztod“8

„Rettung bei plötzlichem Herztod“ verspricht eine Pressemitteilung der Universität Koblenz-Landau. Im Artikel wird eine „App“ für Mobiltelefone vorgestellt, die im Falle eines von plötzlichem Herzstillstand Betroffenen den Weg zum nächsten Defibrillator weisen und erklären soll, was zu tun ist. Warum die Pressemitteilung gerade zu diesem Zeitpunkt erscheint, bleibt offen.

Der Text suggeriert einen lebensrettenden Nutzen, der zwar nicht unplausibel erscheint, aber nach unseren Recherchen nicht nachgewiesen ist. Trotzdem weist der Artikel Leser bereits genau darauf hin, wo die „App“ zu beziehen ist, und dass sie nichts kostet. Was sonst eigentlich Informationen sind, die sich auch im Kriterienkatalog des Medien-Doktors wiederfinden, erhält

8 Bewertung: http://www.medien-doktor.de/pr-watch/2013/05/rettung-bei-plotzlichem-herzstillstand 
hier einen Beigeschmack. Denn wie in einer werblichen Mitteilung unterstreichen die Informationen hier die positiven Eigenschaften des „Produkts“, ohne dass diese wissenschaftlich belegt wären. Für unbedarfte Leser ist das kaum erkennbar, handelt es sich doch um die (wissenschaftliche?) Mitteilung einer Universität. Wirklich wissenschaftlich betrachtet müsste die Behauptung, dass die App den Weg zum Defibrillator so beschleunigt, dass sich mehr Leben retten lassen (der eigentliche Endpunkt!), aber erst - etwa durch Experimente - überprüft werden. Denn vielleicht ist die Bedienung der App ja zu kompliziert oder die Anleitung für die Defibrillation im Praxistest doch kein ausreichender Fortschritt. Solche umfassenden Experimente hat es unseren Recherchen nach aber noch nicht gegeben.

Stattdessen werden die Angaben zum plötzlichen Herzstillstand in der Pressemitteilung dramatisiert - im Sinne des Medien-Doktor-Kriteriums Krankheitsübertreibung („Disease Mongering“). Beschreibungen wie „der Partner, der zuhause unvermutet zusammenbricht“, klingen wie aus dem Leben gegriffen, haben aber kaum einen Bezug zum Auffinden eines Defibrillators im öffentlichen Raum, um den es hier aber geht. Auch die ohne Quelle genannte Zahl von 100.000 Betroffenen mit einem plötzlichen Herzstillstand pro Jahr ist insofern als Übertreibung anzusehen, als es sich um die Gesamtsumme in Deutschland handeln soll (und nicht um die Zahl jener Fälle, die im Einzugsbereich öffentlicher Defibrillatoren verzeichnet wurden). Für eine Marketingabteilung mag das in Teilen vertretbar sein; bei einem Text, der aus einer wissenschaftlichen Standards verpflichteten Institution stammt und in der Bevölkerung ein hohes Vertrauen genießt, sollten andere Kommunikationsstandards gelten.

\section{Fazit}

Die Beispiele und unsere weiteren bisherigen Analysen zeigen, dass die ursprünglich für journalistische Beiträge konzipierten Qualitätskriterien hilfreich sind, Fallstricke und Probleme in Pressemitteilungen zu identifizieren und zu beschreiben. Damit erscheinen sie auch als erste Hilfestellung für Autorinnen und Autoren solcher Mitteilungen geeignet. Angesichts der hier auf das Aufzeigen von Problemen fokussierten Darstellung möchten wir aber betonen: Bei der Bewertung journalistischer Beiträge im Medien-Doktor-Projekt seit 2010 finden wir immer wieder auch Fälle, bei denen ein journalistischer Beitrag hinter den Informationsgehalt der entsprechenden Pressemitteilung zurückfällt: Stellte eine Pressemitteilung vielleicht noch Alternativen zu einer präsentierten OP-Methode vor, ist davon im journalistischen Beitrag nichts mehr zu sehen. Oder: Wird im PR-Text die Finanzierung durch den Lobbyverband klar benannt, kann es durchaus sein, dass diese Information im Zeitungsartikel herausfällt.

Pressestellen können sich jedenfalls weniger denn je darauf verlassen, dass die Mehrzahl der Journalisten Übertreibungen und Fehler korrigiert, fehlende 
6 Von der „Pressemitteilung“ zur „An-alle-Mitteilung“:

Informationen ergänzt und alles zu einem kritischen Gesamtbild zusammenstellt. Zudem sind Pressemitteilungen - wie erwähnt - inzwischen fast immer auch Berichte an eine breite Öffentlichkeit. Sie können Endnutzer online ebenso erreichen wie journalistische Beiträge. Damit erwächst eine ähnliche Verantwortung wie sie von Journalisten (insbesondere im Gesundheitsbereich) abverlangt wird. Die Medien-Doktor-Kriterien sind hier nur ein erster Schritt, können aber unseren bisherigen Analysen nach bereits helfen, dieser Verantwortung besser gerecht zu werden. In welcher Form genau, muss breit diskutiert und auch wissenschaftlich weiter untersucht werden. Wir stellen uns der Diskussion inzwischen an unterschiedlichen Stellen: So konnten wir 2014 etwa bei der Formulierung des Diskussionspapieres der Leitlinien für gute Wissenschaftskommunikation des Siggener Kreises ${ }^{9}$ mitarbeiten, und halfen bei der darauf aufbauenden Arbeitsgruppe, Leitlinien für gute Wissenschafts-PR ${ }^{10} \mathrm{zu}$ formulieren, die im April 2016 zusammen mit einer praxisnahen "Checkliste zur guten Wissenschafts-PR " ${ }^{11}$ offiziell vorgestellt wurden. Ebenso sind wir in der Arbeitsgruppe „Wissenschaft, Öffentlichkeit, Medien“ $(W O ̈ M)^{12}$ der deutschen Akademien vertreten. Auf Konferenzen und Veranstaltungen ${ }^{13}$ stellen wir trotz unserer beschränkten Kapazitäten die Kriterien des Medien-Doktor in Workshops und Vorträgen den Kollegen aus der Wissenschafts-PR vor, testen mit ihnen deren Anwendung und diskutieren mit ihnen über Anpassungen, Korrekturen und Verbesserungen. Seit Juni 2015 haben wir - wie bereits angedeutet - ein neues Format bei unseren Bewertungen auf der Webseite eingeführt: In einem kombinierten Layout präsentieren wir neben der Beurteilung des journalistischen Beitrags auch die Bewertung einer zugehörigen Pressemitteilung. Somit ist es erstmals möglich, beide Texte direkt und Kriterium für Kriterium zu vergleichen. Inzwischen haben auch die amerikanischen Kollegen von HealthNewsReview.org unsere Idee eines „PRWatch“ aufgegriffen: Sie bewerten seit März 2015 nun ihrerseits regelmäßig Bewertungen medizinischer Pressemitteilungen - und finden dabei durchaus auch positive Beispiele.

Wie notwendig dies alles ist, zeigte nicht zuletzt eine Studie im British Medical Journal (BMJ), die das eingangs erwähnte Bauchgefühl von Fiona Fox mit weiteren Daten untermauert: Ein Team um Petroc Sumner und Chris Chambers von der Cardiff University untersuchte 462 Pressemitteilungen von 20 britischen Universitäten, die Fachartikel und die entsprechenden Nach-

9 http://www.wissenschaft-im-dialog.de/ueber-uns/siggener-kreis/

10 Leitlinien zur guten Wissenschafts-PR: https://www.wissenschaft-im-dialog.de/fileadmin/user_upload/Trends_ und_Themen/Dokumente/Leitlinien-gute-Wissenschafts-PR_final.pdf (Abruf 23.11.2016)

11 Checkliste zur guten Wissenschafts-PR: https://www.wissenschaft-im-dialog.de/fileadmin/user_upload/Trends_ und_Themen/Dokumente/Checkliste-gute-Wissenschafts-PR_final.pdf (Abruf 23.11.2016)

$12 \mathrm{http}$ ://www.leopoldina.org/de/politikberatung/arbeitsgruppen/abgeschlossene-arbeitsgruppen/wissenschaftoeffentlichkeit-medien/

13 z.B. 45. Jahrestagung Bundesverband Hochschulkommunikation (September 2014, Konstanz); Jubiläumsveranstaltung „20 Jahre idw“ (Berlin, März 2015), Sitzung des AK Presse der Leibniz-Gemeinschaf (Juni 2015, Göttingen) 
richtenartikel (Sumner et al. 2014). Das Ergebnis: Gut jede dritte Pressemitteilung wies in mehrfacher Hinsicht Übertreibungen auf.

\section{Kriterien zur Bewertung medizinjournalistischer Berichterstattung}

Mit 13 (zehn „medizinjournalistischen“ und drei „allgemeinjournalistischen") Kriterien bewerten wir die Berichterstattung über Medikamente, Verfahren, Therapien und diagnostische Tests. Zugleich empfehlen wir Journalisten diese als „Recherchegeländer" für das Produzieren ihrer Beiträge. In ähnlicher Form können diese Kriterien Hilfestellung beim Schreiben von Pressemitteilungen über solche Themen sein. Das untersuchen wir derzeit auch in einem Forschungsprojekt (2013-2016), das vom BMBF finanziert wird.

1. Beschreiben Sie wie groß oder klein der mögliche Nutzen/das Studienergebnis in konkreten Zahlen ist, nicht nur in relativen Angaben, sondern auch in absoluten. Ist eine Aussage zum Nutzen noch nicht möglich, machen Sie das deutlich und vermeiden Sie Spekulationen.

2. Gehen Sie ebenso ausführlich auf das Thema möglicher Risiken und Nebenwirkungen ein und quantifizieren Sie auch diese soweit möglich.

3. Erklären Sie Leserinnen und Lesern (Zuhörern, Zuschauern) wie aussagekräftig eine Studie ist, welche Stärken und welche Schwächen sie hat. Oder vermitteln sie, wie gut Aussagen zu Nutzen und Risiken durch Studien belegt sind (oder auch nicht), wenn Sie die Öffentlichkeit über eine Intervention (z.B. ein neues OP-Verfahren an einer Klinik) informieren.

4. Holen Sie die Einschätzung einer unabhängigen/unbeteiligten Quelle ein und geben Sie Informationen zur Finanzierung der Studie und möglichen Interessenkonflikten. Auch die Berücksichtigung eines kommentierenden Editorials in einer Fachzeitschrift kann sich hier eignen.

5. Eine Pressemitteilung sollte eher Anlass als Quelle sein, als Hauptquelle ist sie nicht ausreichend. Mitunter haben nicht einmal die Verfasser einer Pressemitteilung die Originalstudie gelesen.

6. Erklären Sie, was das Neue an der Studie oder Intervention ist oder ob diese bereits lange bekannt ist. Machen Sie auch deutlich, wenn ein Wirkstoff bereits zur Therapie anderer Erkrankungen eingesetzt wurde.

7. Weisen Sie auf Alternativen zur vorgestellten Therapie, zum vorgestellten Medikament oder OP-Verfahren hin oder machen Sie klar, wenn es keine gibt. Auch eine Intervention zu unterlassen, kann eine gleichwertige Alternative sein, weil die Abwägung von Nutzen und Risiken sehr persönliche Entscheidungen sind (z.B. bei Früherkennungsmaßnahmen).

8. Machen Sie deutlich, ab wann und wo die Intervention (in der Region, überregional, weltweit) unter welchen Umständen (nur innerhalb einer Studie oder bei jedem Hausarzt oder Apotheker?) verfügbar ist. 
6 Von der „Pressemitteilung“ zur „An-alle-Mitteilung“:

Der Medien-Doktor PR Watch an der TU Dortmund

9. Geben Sie Informationen zu den konkreten Kosten für Betroffene (auch Übernahme durch Krankenkassen? IGeL?) oder für die Gesellschaft, wenn diese absehbar oder bekannt sind.

10. Vermeiden Sie Dramatisierungen und Übertreibungen einer Erkrankung und stellen sie Ersatzparameter (physiologische Messwerte wie Cholesterinspiegel, Blutdruck usw.) nicht bereits für sich genommen als Krankheit dar.

11. Wählen Sie ein Thema aus, das wirklich relevant (z.B. durch die Veröffentlichung einer Studie oder das aktuelle Nachrichtengeschehen) oder zumindest ungewöhnlich ist; berichten Sie dem Medium entsprechend zeitnah und erklären, wann z.B. eine Studie veröffentlich wurde.

12. Achten Sie auf eine verständliche Darstellung (wenig Fremdworte usw.) und einfache, wenn möglich auch abwechslungsreiche Vermittlung (z.B. Dramaturgie) des Themas.

13. Vermeiden Sie Faktenfehler und geben Sie Quellen an zu Daten und Zahlen, die sie nennen.

\section{Quellenangaben}

Fox F: Dodgy science headlines: PR can share the blame; in: On science in the media. Unter http://fionafox. blogspot.de/2012_02_01_archive.html, 6.2.2012, zuletzt aufgerufen am 4.1.2015

Prasad V, Jorgenson I, Ioannidis I, Cifu A: Observational studies often make clinical practice recommendations: an empirical evaluation of authors' attitudes; in I Clin Epidemiol, 2013, 66:4, 361-366.e4 (DOI: 10.1016/j. jclinepi.2012.11.005)

Reifegerste D, Oelschlägel F, Schumacher M: „Copy Factories“ im Gesundheitsjournalismus? Medienresonanzanalyse einer Krankenkasse; in: Qualität im Gesundheitsjournalismus - Perspektiven aus Wissenschaft und Praxis; Hrsg: Lilienthal V, Reineck D, Schnedler T; Wiesbaden 2014, S. 159-172 (D0I: 10.1007/978-3658-02427-7)

Schwartz L, Woloshin S, Andrews A, Stukel T: Influence of medical journal press releases on the quality of associated newspaper coverage: retrospective cohort study; in BM), 2012; 344:jan27 1, d8164 (D0I: 10.1136/ bmj.d8164)

Sumner P, Vivian-Griffiths S, Boivin I, Williams A, Venetis C, Davies A, Ogden I, Whelan L, Hughes B, Dalton B, Boy $\mathrm{F}$, Chambers $\mathrm{C}$ : The association between exaggeration in health related science news and academic press releases: retrospective observational study; in BM), 2014;349:dec9 7, g7015 (D0I: 10.1136/bmj.g7015)

Yavchitz A, Boutron I, Bafeta A, Marroun I, Charles P, Mantz I, Ravaud P: Misrepresentation of Randomized Controlled Trials in Press Releases and News Coverage: A Cohort Study, in PLoS Medicine 2012, 9(9): e1001308 (DOI: 10.1371/journal.pmed.1001308).

\section{Weiterführende Literatur}

Weingart P, Patricia S (Hrsg.): Wissen - Nachricht - Sensation. Zur Kommunikation zwischen Wissenschaft, Öffentlichkeit und Medien; 424 Seiten (2014)

Wormer H, Anhäuser M: „Gute Besserung!“ - und wie man diese erreichen könnte. Erfahrungen aus drei Jahren Qualitätsmonitoring Medizinjournalismus auf medien-doktor.de und Konsequenzen für die journalistische Praxis, Ausbildung sowie Wissenschafts-PR; Qualität im Gesundheitsjournalismus - Perspektiven aus Wissenschaft und Praxis; Hrsg.: Lilienthal V, Reineck D, Schnedler T; Wiesbaden 2014, S. 159-172 (D0I 10.1007/978-3-658-02427-7) 